ISSN 1014-4874

DOI : http://dx.doi.org/10.4314/rj.v27i1.1

\title{
Simulation of a Shielded Thermocouple
}

\author{
Fredrik Berntsson ${ }^{1}$, Fidèle Ndahayo, Yves Nyalihama and, \\ Jean Marie Vianney Munyeshyaka ${ }^{2}$ \\ ${ }^{1}$ Linköping University, S-581 83, Linköping, Sweden. \\ ${ }^{2}$ National University of Rwanda, Box 117, Butare, Rwanda.
}

\begin{abstract}
A shielded thermocouple is a measurement device used for monitoring the temperature in chemically, or mechanically, hostile environments. The sensitive parts of the thermocouple are protected by a shielding layer. In this work we use numerical methods to study the accuracy and dynamic properties of a shielded thermocouple design. Also, we show that by formulating and solving an appropriate inverse problem, we can significantly reduce the errors in the measurement process.
\end{abstract}

\section{Introduction}

Temperature measurements in mechanically, or chemically, hostile environments are often difficult to perform since the recording device can be destroyed. In such situations a shielded thermocouple can be used [6, 10]. In such a device the measurement points, as well as wires, are protected by a shielding material.

Although shielding material protects the measurement points and the wires, it can also reduce the accuracy and change dynamic properties of the measurement device. In the stationary case there may be a thermal gradient along the length of the thermocouple acting as a heat sink lowering the temperature locally. Also if the temperature of the surrounding medium changes it will take some time until the change has propagated through the shielding material and becomes notice- able at the measurement points. These effects cannot be avoided but can be minimized by good thermocouple design. A typical shielded thermocouple is displayed in Figure 1.1.

In this work we attempt to use numerical methods to investigate the performance of shielded thermocouple designs. A mathematical model of the thermocouple is obtained by derivation of the heat propagation equation in cylindrical coordinates and by considering the axial symmetry of the problem [7]:

The unknown temperature function $T(r, t)$ is the solution of the equation 


$$
\frac{1}{r}\left(k(r) r T_{r}\right)_{r}-\mu(r)\left(T-T_{\infty}\right)=c_{p}(r) \rho(r) T_{t}, 0<r<r_{2}
$$

which satisfies boundary conditions,

$$
T_{r}(0, t)=0 \text { and } T\left(r_{2}, t\right)=F(t) .
$$

Here $k$ is the thermal conductivity, $c_{p}$ is the specific heat capacity, $\rho$ is the density, $T_{\infty}$ is the ambient temperature, and $\mu$ describes the conductance of heat along the thermocouple. The function $F(t)$ is the time dependent temperature of the medium surrounding the thermocouple, or the temperature that we want to measure. By solving (1.1) we can compute the temperatures at the measurement points, i.e. at $r=r_{0}$ and $r=r_{1}$.
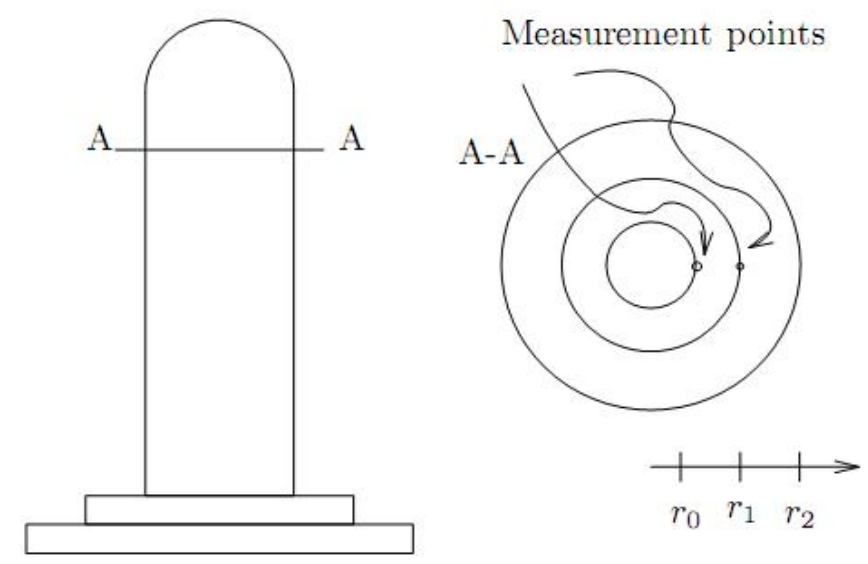

Figure 1.1 : A sketch of a shielded thermocouple. The measurement points are protected by a layer of magnesium-oxide. The hollow interior $\left(0<r<r_{0}\right)$ contains wires.

Solving the forward problem (1.1) gives us important information, such as the step function res- ponse for a specific shielded thermocouple design. In this work we will further investigate the possibility of using the recorded temperatures, at $r=r_{0}$ and $r=r_{1}$ respectively, to compute the temperature at the surface $r=r_{2}$. Calculating the surface temperature of a body given interior measurements is an inverse problem that has been studied extensively. The problem is ill-posed as small measurement errors can be blown up and dominates the numerical solution. Hence special regularization techniques are needed. 
Recall that the temperature $T(r, t)$ is recorded by thermocouple giving us access to measurements,

$$
T\left(r_{0}, t\right)=G(t) \text { and } T\left(r_{1}, t\right)=H(t)
$$

By solving a well-posed problem in the interval $r_{0}<r<r_{1}$, the heat-flux $T_{r}\left(r_{1}, t\right)$ can be computed. Hence the inverse problem is: Find the temperature $T(r, t)$ that satisfies

$$
\frac{1}{r}\left(k(r) r T_{r}\right)_{r}-\mu(r)\left(T-T_{\infty}\right)=c_{p}(r) \rho(r) T_{t}, r_{1}<r<r_{2}
$$

given Cauchy data,

$$
T\left(r_{1}, t\right)=H(t) \text { and } T_{r}\left(r_{1}, t\right)=q(t)
$$

The paper is organized as follows. The ill-posedness of the inverse problem is demonstrated in Section 2. Numerical algorithms for solving the equations (1.1) and (1.4) are discussed in Sec- tion 3. In Section 4 a few numerical experiments are presented and, finally, some conclusions are given in Section 5.

\section{Ill--posedness and Regularization}

In this section we study the inverse problem (1.4) and show that it is illposed $[1,2,4]$. We cons-ider the simplified problem: Find the temperature $T(x, t)$ satisfying:

$$
\left\{\begin{array}{cl}
k T_{x x}=T_{t}, & x \geq 0 \quad 0<t<\infty \\
T(x, 0)=0, & x \geq 0 \\
T(1, t)=g(t),\left.T\right|_{x \rightarrow \infty} \text { bounded } &
\end{array}\right.
$$

where $x$ is the space coordinate, $t$ is time, $g(t)$ is the recorded temperature at the measurement point $x=1, k$ is the thermal diffusivity. Of course, since $g(t)$ is assumed to be measured, there will be measurement errors, and we will actually have a data function $g_{m} \in L^{2}$ satisfying, $\quad\left\|g_{m}-g\right\|=\left\|g_{m}-T(1,).\right\|<\varepsilon$

where $\varepsilon>0$ represents a bound on the measurements error.

The problem (2.1) is ill-posed in the sense that the solution, if it exists, does not depend continuously on the data. The ill-posedness can be seen by solving the problem in the Fourier domain. In order to simplify the analysis, we define all functions to be zero for $t<0$. 
Let

$$
\hat{g}(\xi)=\frac{1}{\sqrt{2 \pi}} \int_{-\infty}^{\infty} g(t) e^{-i \xi t} d t
$$

be the Fourier transform of the exact data. The problem (2.1) can now be formulated in frequency space, as follows:

$$
\left\{\begin{array}{c}
k \hat{T}_{x x}(x, \xi)=i \xi \hat{T}(x, \xi), \quad x \geq 0, \quad-\infty<\xi<\infty \\
\hat{T}(1, t)=\hat{g}(\xi), \quad-\infty<\xi<\infty \\
\left.T\right|_{x \rightarrow \infty} \text { bounded },
\end{array}\right.
$$

The solution to the above problem is given by

$$
\hat{T}(x, \xi)=e^{\sqrt{\frac{i \xi}{k}}(1-x)} g(\xi)
$$

where $\sqrt{i \xi}$ denotes the principal value of the square root. Since the real part of $\sqrt{i \xi}$ is positive, and our solution $\hat{T}(x, \xi)$ is assumed to be in $L^{2}(I R)$, we see that the exact data function $\hat{g}(\xi)$, must decay rapidly as $|\xi| \rightarrow+\infty$. Now we assume that the measured data function satisfies $g_{m}(t)=g(t)+\sigma(t)$, where $\sigma \in L^{2}(I R)$ is a small measurement error. If we try to solve the problem using $g_{m}$ as data we get a solution

$$
\hat{u}(x, \xi)=e^{\sqrt{\frac{i \xi}{k}}(1-x)}(\hat{g}(\xi)+\hat{\sigma}(\xi))=\hat{T}(x, \xi)+e^{\sqrt{\frac{i \xi}{k}}(1-x)} \hat{\sigma}(\xi)
$$

Since we cannot expect the error $\hat{\sigma}(\xi)$ to have the same decay in the frequency as the exact data $\hat{g}(\xi)$ the solution $\hat{u}(x, \xi)$ will not, in general be in $L^{2}(I R)$. If we try to solve the problem numerically, high frequency components in $\sigma$, are magnified and can destroy the solution.

In our work we stabilize the problem by introducing a cut-off frequency $\xi_{c}$, and by removing high frequencies from the solution $\hat{T}(x, \xi)$. This regularization technique works rather well and a complete analysis of the simplified case presented above can be found in $[1,2]$.

The more complicated equation (1.4) is also ill-posed and a similar analysis can be carried out in the frequency domain, see [5]. The analysis shows that the problem can be regularized by cutting off high frequencies in the solution and that the parameter choice rule, that can be used for finding a good value for the cut-off frequency, can be derived. 


\section{Numerical Algorithms}

The forward problem (1.1) is a regular well-posed boundary value problem for a parabolic equa- tion and hence a standard finite difference method can be used for solving it. In our codes we introduce a space discretization $\left\{r_{i}\right\}_{i=0}^{N-1}$ and write $T(:, t)=\left(T\left(r_{0}, t\right), \ldots, T\left(r_{N-1}, t\right)\right)^{T}$. We discretize the governing equation using central differences and obtain a linear system

$$
A T(:, t)+b(t)=T_{t}(:, t)
$$

The Crank-Nicholsson method is used to deal with the time dependence and in each step of our numerical scheme we need to solve a (sparse) linear system,

$$
\left(I-\frac{d t}{2} A\right) T(:, j)=\left(I+\frac{d t}{2} A\right) T(:, j-1)+\frac{1}{2}(b(j)+b(j-1))
$$

The Crank--Nicholsson method is accurate, efficient, and works well for our purposes. Using the above scheme we create simulated experiments by picking a surface temperature $T\left(r_{2}, t\right)=F(t)$, and computing the corresponding temperature histories at the measurement points.

The above Crank-Nicholsson discretization can be described as a timemarching scheme. For the inverse problem (1.4) we will instead use a space-marching algorithm. For our numerical sche- me we introduce a time grid $\left\{t_{j}\right\}_{j=0}^{M-1}$, and denote by $T=\left(T\left(r, t_{0}\right), \ldots, T\left(r, t_{M-1}\right)\right)^{T}$. The governing equation (1.4) can be written as a system of of ordinary differential equations,

$$
\left(\begin{array}{c}
T \\
r k(r) T_{r}
\end{array}\right)_{r}=\left(\begin{array}{cc}
0 & \frac{1}{k(r) r} I \\
\mu(r) I+r \rho(r) c_{p}(r) D & 0
\end{array}\right)\left(\begin{array}{c}
T \\
r k(r) T_{r}
\end{array}\right)+\left(\begin{array}{c}
0 \\
-\mu(r) T_{\infty}
\end{array}\right),
$$

with initial-boundary values

$$
T\left(r_{0}, t\right)=G_{m}(t), T_{r}\left(r_{0}, t\right)=L_{m}(t) \text {, and } T(r, 0)=0, t \in I R .
$$

The matrix $D$ represents a discretization of the time derivative $\partial_{t}$.

As discussed previously the inverse problem is ill-posed $[1,2]$ and hence regularization is needed. In our method the problem is stabilized by computing the time derivative by using a truncated discrete Fourier transform. Thus the matrix $D$ is taken as,

$$
D \equiv D_{c}=F \Lambda_{c} F^{H}
$$


where $F$ is the Fourier matrix and $\Lambda_{c}$ is the diagonal matrix corresponding to differentiation of the trigonometric interpolant in which frequency components with $|\xi|>\xi_{c}$ are explicitly set to zero. The cut-off frequency $\xi_{c}$ is a parameter that can be used to control the smoothness of the solution to the inverse problem, and represents a trade-off between accuracy and the influence of measurement errors.

The system of ODEs (3.1) can be solved using standard methods. For our numerical code we use an explicit solver of Runge-Kutta type, e.g. Matlab ode45.

\section{Simulated Experiments}

In this section we present experiments intended to demonstrate the usefulness of numerical simulations for investigating the performance of shielded thermocouple designs. Also, we will show that the accuracy of the measurements can be improved by solving the corresponding inverse problem.

During the tests we will assume that the shielding layers of the thermocouple have dimensions $r_{2}=10 \mathrm{~mm}, r_{1}=8 \mathrm{~mm}$, and $r_{0}=4 \mathrm{~mm}$. Further, we assume that the measurements take place during $2000 \mathrm{~s}$. The material parameters are chosen to be realistic. We use the expressions for material constants for porcelain taken from [6].

Firstly we pick an appropriate function $F(t)$ (the surface temperature) and simulate measure- ments $G(t)$ and $H(t)$ by solving the direct problem. The results for two different functions $F(t)$ are displayed in Figure 4.1. Two important effects can be seen. Firstly a change of temperature at the surface of the thermocouple must propagate through the shielding material before it is noticeable at the measurement points. Hence there is a time delay in the measurement device that depends on both dimensions and material choices. Also there is an asymptotic error because the interior of the shielded thermocouple acts as a heat sink. These two effects depend on the thermocouple design and material choices for the shielding layers. 

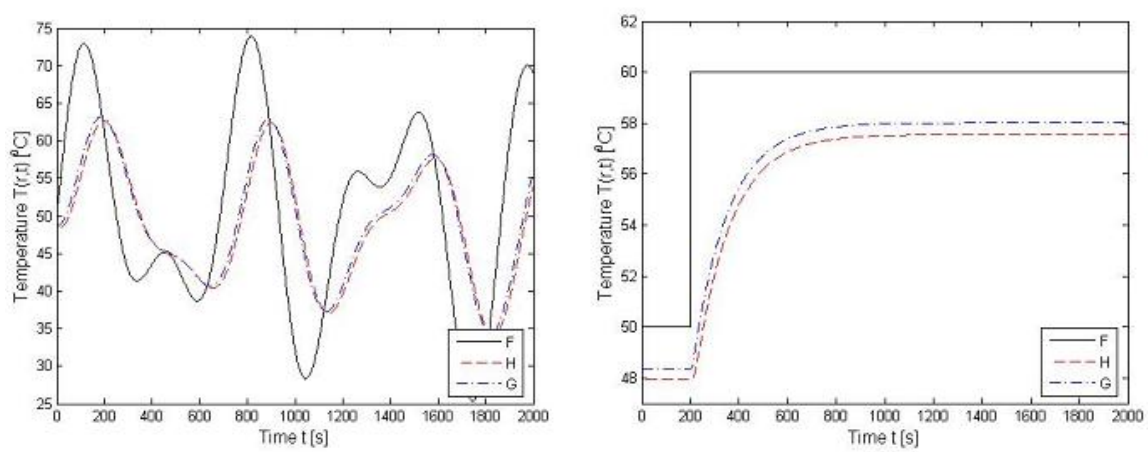

Figure 4.1: The temperature response at the measurement points $T\left(r_{1}, t\right)=G(t)$ (dash-dotted curve) and $T\left(r_{0}, t\right)=H(t)$ (dashed curve) given the surface temperature $T\left(r_{2}, t\right)=F(t)$ (solid curve). We present the case where $F(t)$ is a smooth function (left) and a step function (right).

The time delay and asymptotic error are unavoidable when using a shielded Thermocouple [6]. However these effects can be compensated for by solving an inverse problem. For the numerical tests we created simulated measurements by solving the direct problem; and adding normally distributed noise with variance $10^{-1}$ giving us data vectors $G_{m}$ and $H_{m}$.

Firstly we selected a step function $F(t)$ and created the simulated measurements $G_{m}$ and $H_{m}$. By solving the inverse problem we find an approximation $F_{m}$ of the surface temperature. The noisy data and computational results obtained using different cut-off frequencies $\xi_{c}$ are display- ed in Figure 4.2. We see that it is possible to compensate for both the time delay and to reduce the asymptotic error considerably. The effects of the regularization parameter $\xi_{c}$ can also be seen.
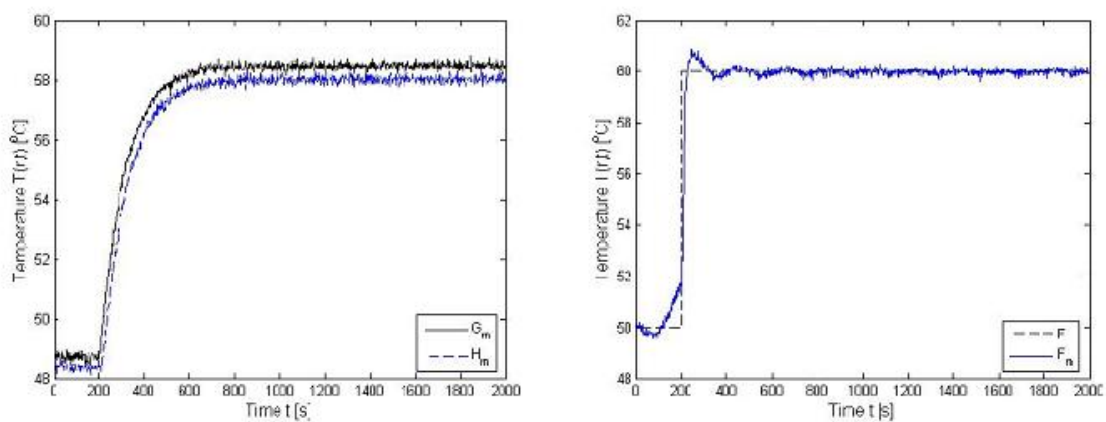

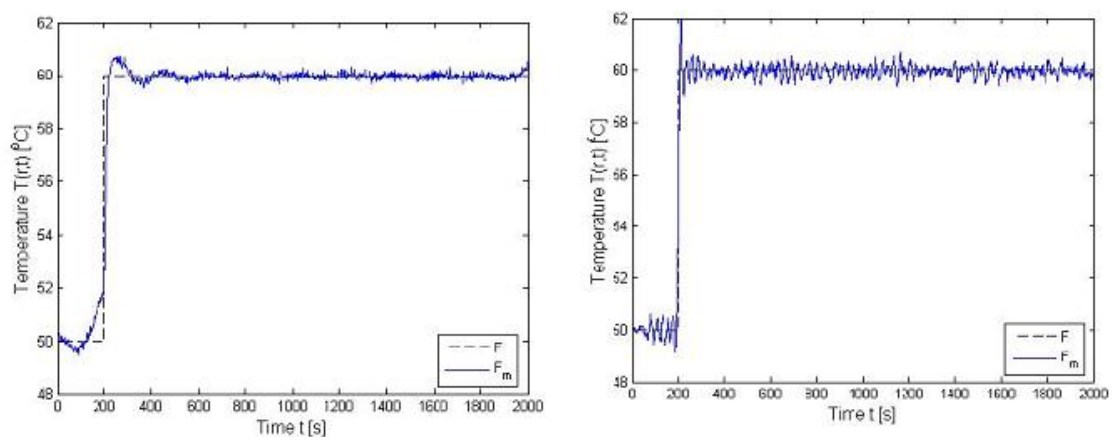

Figure 4.2: The simulated noisy data $H_{m}$ and $G_{m}$ (top, left). Also the approximate surface temperature $F_{m}$ obtained using $\xi_{c}=20$ (top, right), $\xi_{c}=20$ (bottom, left), and $\xi_{c}=200$ (bottom, right).

\section{Concluding Remarks}

In this work we have showed the usefulness of numerical models for understanding the performance of a shielded thermocouple. Firstly, numerical simulations have been used for simulating such a device and for understanding how the thermocouple design influences the accuracy of the measurements. Two effects have been studied in particular: the time delay since the thermal signal needs to propagate through the shielding material and the asymptotic error resulting from the measurement device conducting heat away from the medium.

Secondly, the inverse problem of finding the "true" temperature of the medium given the temperature histories at the two measurement points is ill-posed but using an appropriate regularization scheme an accurate solution can still be found. By using the inverse problem solver as a data filtering device we can remove the asymptotic error and reduce the effects of the time delay.

Numerical experiments (not reported in this paper) demonstrate that compensating for the asymptotic error is easy and the strength of the effects causing the asymptotic error has little impact on the degree of ill-posedness of the inverse problem. The time delay is related to the smoothing property of the direct problem and is directly related to the ill-posedness of the inverse problem. 


\section{References}

[1] L. Eldén, F. Berntsson, and T. Reginska, Wavelet and Fourier methods for solving the sideways heat equation, SIAM J.Sci. Comput. 21(6) : 287-2205, 2000.

[2] F. Berntsson, A spectral method for solving the sideways heat equation inverse problem, Vol.15, pp 891-906, August 1999.

[3] F. Berntsson, and L. Eldén, An inverse heat conduction problem and an application to heat treatment of aluminium, In M.Tanaka and G.S Dulikravich editors, Inverse problem in Engineering Mechanics II. International symposium on inverse problems, Engineering Mechanics 2000(ISIP 2000), Nagano, Japan pp.99-106.Elsevier 2000.

[4] F. Berntsson, Numerical methods for solving a non-characteristic Cauchy problem for a parabolic equation, Technical report LITHMAT-R-2001-17 September 2001.

[5] Yves Nyalihama and Jean Marie Vianney Munyeshyaka. Improving Shielded Thermocouple measurements. Masters Thesis, National University of Rwanda, Butare, Rwanda.

[6] Xlu, P. Tervola and M. Viljanen, An efficient analytical solution to transcient heat conduction in a one-dimensional hollow composite cylinder, Math. Gen. 38(2005) 10145-10155.

[7] Gunnar Andersson, Karl Storck. Matts Karlsson, and Dan Loyd, Shielded thermocouples for Dynamic temperature measurements- $A$ heat transfer analysis. Tenth international conference for numerical methods in thermal problems, Swansea, UK, July 21-25 1997 pp 593603.

[8] Teresa Reginska. Application of wavelet shrinkage to solving the sideways heat equation. BIT 2001, vol 41 No 5 pp 1101-1110.

[9] A. K Tikhe and K.C Deshmukh. Inverse heat conduction problem in a Thin circular plate and its thermal deflection, the Korean society for industrial and applied Mathematics, 2005.

[10] Linda G.Blevins, and William M.Pitts. Modeling of bare and a spirated thermocouples in compartment fires. Fire safety journal 33(1999) 239-259. Elsevier.

[11] Per Christian Hansen. Rank-deficient and discrete ill-posed problems, SIAM, 1998. 